\title{
PAPER \\ Spectral Efficiency of Fundamental Cooperative Relaying in Interference-Limited Environments
}

\author{
Koji YAMAMOTO $^{\dagger a)}$, Member, Hirofumi MARUYAMA ${ }^{\dagger}$, Takashi SHIMIZU $^{\dagger}$, Student Members, $^{\circ}$ \\ Hidekazu MURATA ${ }^{\dagger}$, Member, and Susumu YOSHIDA ${ }^{\dagger}$, Fellow
}

\begin{abstract}
SUMMARY The spectral efficiency of cooperative relaying in interference-limited environments in which a given channel is spatially reused is investigated. Cooperative relaying is a promising technique that uses neighboring stations to forward the data toward the destination in order to achieve spatial diversity gain. It has been reported that by introducing cooperative relaying into communication between an isolated sourcedestination pair, the error rate or spectral efficiency is generally improved. However, it is not intuitively clear whether cooperative relaying can improve the performance in interference-limited environments because the simultaneous transmission of multiple stations increases the number of interference signals. Assuming the most fundamental cooperative relaying arrangement, which consists of only one relay station, numerical results reveal that cooperative relaying is not always superior to non-cooperative single-hop and two-hop transmissions in terms of spectral efficiency.

key words: cooperative relaying, multihop, area spectral efficiency, bandwidth efficiency
\end{abstract}

\section{Introduction}

Recent advancements in radio technologies and the success of the Internet have shown the potential of information systems as ubiquitous networks that are accessible from anywhere, at any time, and with any device [1]. A key enabling technology for such networks is multihop transmission. By using multihop transmission, signals are forwarded from a source to its intended destination, which can be relatively distant [2]. For this type of wide-area radio network, referred to as a multihop radio network, high spectral efficiency is required because the amount of spectrum available for wireless systems is limited. A new technique to enhance the spectral efficiency of multihop radio networks is cooperative relaying [3]-[8].

In cooperative relaying, the source, destination, and relay stations share their resources to forward the data, which achieves spatial diversity gain against fading. The performance of cooperative relaying has mainly been evaluated in terms of error rate or spectral efficiency assuming a singleisolated communication [6]-[8] or two pairs of communications [3], [5]. It has been reported that the introduction of cooperative relaying reduces the error rate and enhances the spectral efficiency. In particular, it has been reported that cooperative relaying outperforms non-cooperative multihop transmission in terms of spectral efficiency.

\footnotetext{
Manuscript received August 31, 2007.

Manuscript revised March 26, 2008.

The authors are with the Graduate School of Informatics, Kyoto University, Kyoto-shi, 606-8501 Japan.

a) E-mail: contact-h20@hanase.kuee.kyoto-u.ac.jp DOI: 10.1093/ietcom/e91-b.8.2674
}

One of the remaining issues in cooperative relaying is its introduction to radio communication systems, in which co-channel interference exists. It is not intuitively clear whether such cooperative relaying can improve the spectral efficiency in interference-limited environments as effectively as is expected in noise-limited environments. This is because in some cooperative relaying systems, more than one station may transmit simultaneously in order to achieve diversity gain. Simultaneous transmission inherently increases the number of co-channel interference sources, as compared to non-cooperative two-hop transmission, and it may be impossible to achieve a significant improvement, as is expected in isolated environments.

Assuming a simple but fundamental cooperative relaying arrangement, which consists of only one relay station between a source and a destination, as described in [6], the present paper introduces a meaningful theoretical framework for analyzing two types of spectral efficiency for cooperative relaying systems: bandwidth efficiency (BE) and area spectral efficiency (ASE) in interference-limited environments. $\mathrm{BE}$ is defined to be the maximum end-to-end bit rate through multiple hops per unit bandwidth. ASE is defined to be the BE per unit area as defined in cellular networks [9]-[11].

The remainder of the present paper is organized as follows. In Sect. 2, we discuss a system model and introduce cooperative relaying. In Sect. 3 , we derive and evaluate the $\mathrm{BE}$ in interference-limited environments while considering the BE in noise-limited environments [6]. In Sect. 4, we investigate the ASE of cooperative relaying systems. Section 5 concludes the present paper with a summary and some final remarks.

\section{System Model}

\subsection{General Assumptions}

Consider a cooperative relaying system with only one relay candidate $(R)$ located between a given source $(S)$ and destination (D), as illustrated in Fig. 1(a). Each station is equipped with an omnidirectional antenna and is assumed to be able to either transmit or receive at a given time.

\subsection{Interference-Limited Environments}

We assume interference-limited environments in which co- 


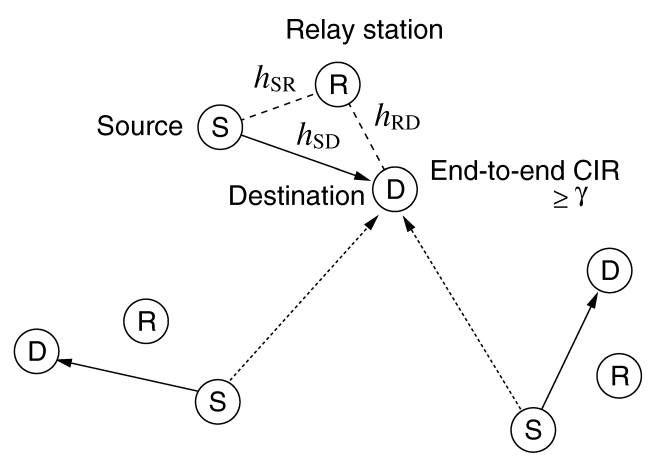

(a) Model of relay channels.

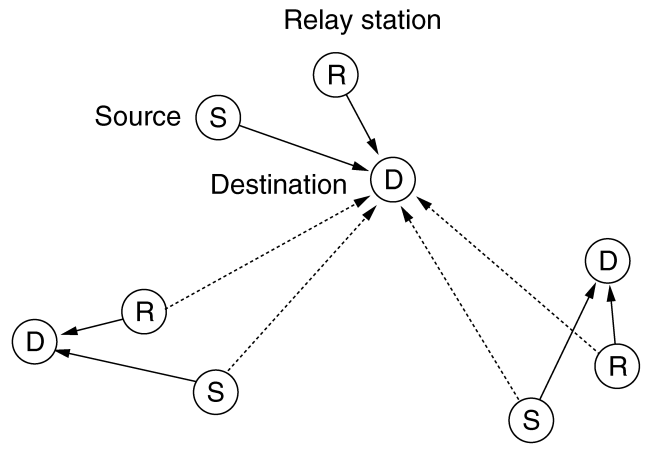

(b) The number of interference signals is doubled due to simultaneous transmission by the source and the relay station when using cooperative relaying $(\mathrm{RD}, \mathrm{SR})$ and $(\mathrm{R}$, SR) in slot 2 .

Fig. 1 Solid arrows represent desired signals and dashed arrows represent co-channel interference.

channel interference is dominate over the thermal noise. All interference signals are assumed to be equivalent to Gaussian noise over the channel, as in [12] (which is discussed in more detail in [10]).

In order to represent the density of the simultaneous communication, we introduce the end-to-end carrier to interference ratio (ECIR) as follows. Consider the situation in which every source transmits directly to a designated destination regardless of whether each source-destination pair can communicate directly, as shown in Fig. 1(a), and we designate the received carrier-to-interference ratio (CIR) as being the ECIR. This ECIR is considered to be an appropriate parameter to reflect the density of the simultaneous communication since the absolute value of this density does not have meaning in the following simple model (which is discussed in more detail in [13]). We first evaluate the BE using the ECIR as a parameter. We then show the relationship between ASE and BE, which does not depend on the ECIR.

The spectral efficiency of cooperative relaying is sensitive to several factors, particularly fairness [14], [15]. Since the main purpose of this investigation is to examine the impact of cooperative relaying on the spectral efficiency of wireless networks, we set aside the fairness problem for now. Thus, we investigate the spectral efficiency under the condition that all communication at a given time has almost

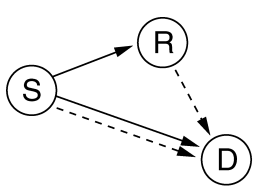

(a) $(\mathrm{RD}, \mathrm{SR})$.

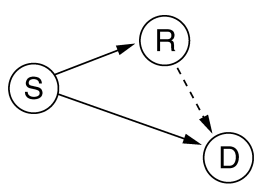

(b) $(\mathrm{RD}, \mathrm{R})$

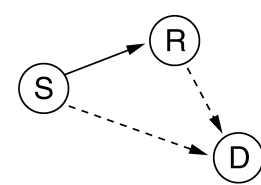

(c) $(\mathrm{R}, \mathrm{SR})$.

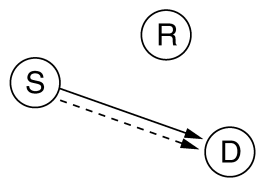

(d) Single-hop.

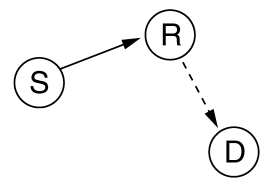

(e) Two-hop $=(\mathrm{R}, \mathrm{R})$
Fig. 2 Three cooperative and two non-cooperative schemes. Solid arrows represent transmissions in slot 1 and dashed arrows represent transmissions in slot 2

the same condition, i.e., communication methods for all transmissions are the same and ECIR is larger than $\gamma$. In addition, the interference power from the source and that from the designated relay station are assumed to be the same.

\subsection{Cooperative Relaying}

One technique to enable forwarding of a signal under the above assumptions is dividing the spectrum into different orthogonal time slots and allocating these slots to each transmitting station [6]. Time slots are assumed not to be synchronized among all routes.

The relay station is assumed to decode and forward the signal received from the source to the destination using two time slots and let $i(=1,2)$ denote the index of the slot. We assume the following three cooperative schemes (RD, SR), (RD, R), and (R, SR) and two non-cooperative schemes, as shown in Fig. 2, where for example (RD, SR) denotes that both $R$ and $D$ receive in slot 1 and both $S$ and $R$ transmit in slot 2 .

(RD, SR) This scheme is proposed in [6]. In slot 1, both the relay station and the destination receive the signal from the source. In slot 2 , both the source and the relay station transmit to the destination.

$(\mathbf{R D}, \mathbf{R})$ This scheme is proposed in [5], [16]. In slot 1, both the relay station and the destination receive the signal from the source. In slot 2 , only the relay station transmits to the destination.

(R, SR) This scheme is also proposed in [5], [16]. In slot 1 , only the relay station receives the signal from the source. In slot 2 , both the source and the relay station transmit to the destination.

Single-hop In slots 1 and 2, the source transmits directly to the destination.

Two-hop In slot 1, only the relay station receives the signal from the source. In slot 2, only relay station transmits to the destination. 


\section{Bandwidth Efficiency}

\subsection{Analysis}

In this section, we modify the performance expressions of cooperative relaying in [6] in order to investigate the effect of co-channel interference.

\subsubsection{Cooperative Relaying (RD, SR)}

The signal transmitted by the source in slot $i$ is denoted as $x_{i}$. Let $I_{i}$ denote the interference power in slot $i$. The signal received by the relay station in slot 1 is then given by

$$
y_{\mathrm{R}, 1}=\sqrt{E_{\mathrm{SR}}} h_{\mathrm{SR}} x_{1}+n_{\mathrm{R}, 1}
$$

where $E_{\mathrm{SR}}$ denotes the average signal energy at the relay station, $h_{\mathrm{SR}}$ denotes a complex unit-power channel gain, and $n_{\mathrm{R}, 1} \sim \mathcal{C N}\left(0, I_{1}\right)$ is interference treated as Gaussian noise process in slot 1 . Similar to (1), the signals received by the destination in slots 1 and 2 are given by

$$
\begin{aligned}
& y_{\mathrm{D}, 1}=\sqrt{E_{\mathrm{SD}}} h_{\mathrm{SD}} x_{1}+n_{\mathrm{D}, 1} \\
& y_{\mathrm{D}, 2}=\sqrt{E_{\mathrm{SD}}} h_{\mathrm{SD}} x_{2}+\sqrt{E_{\mathrm{RD}}} h_{\mathrm{RD}} x_{1}+n_{\mathrm{D}, 2}
\end{aligned}
$$

where $E_{\mathrm{SD}}$ and $E_{\mathrm{RD}}$ denote the average signal energy at the destination through S-D and R-D links, $h_{\mathrm{SD}}$ and $h_{\mathrm{RD}}$ denote complex unit-power channel gains through S-D and R-D links, and $n_{\mathrm{D}, i} \sim \mathcal{C N}\left(0, I_{i}\right)$ is interference treated as Gaussian noise process in slot $i$.

Let $I$ denote the interference power when every source transmits directly to a designated destination as shown in Fig. 1(a). Then the average received ECIR can be written as $\gamma=E_{\mathrm{SD}} / I$. In addition, let $\lambda_{i}=I / I_{i}, \mu=E_{\mathrm{SR}} / E_{\mathrm{SD}}$, and $v=E_{\mathrm{RD}} / E_{\mathrm{SD}}$, we can rewrite (2) as

$$
\begin{aligned}
& {\left[\begin{array}{l}
\sqrt{\lambda_{1}} y_{\mathrm{D}, 1} \\
\sqrt{\lambda_{2}} y_{\mathrm{D}, 2}
\end{array}\right]} \\
& =\left[\begin{array}{cc}
\sqrt{E_{\mathrm{SD}} \lambda_{1}} h_{\mathrm{SD}} & 0 \\
\sqrt{E_{\mathrm{RD}} \lambda_{2}} h_{\mathrm{RD}} & \sqrt{E_{\mathrm{SD}} \lambda_{2}} h_{\mathrm{SD}}
\end{array}\right]\left[\begin{array}{l}
x_{1} \\
x_{2}
\end{array}\right]+\left[\begin{array}{c}
\sqrt{\lambda_{1}} n_{\mathrm{D}, 1} \\
\sqrt{\lambda_{2}} n_{\mathrm{D}, 2}
\end{array}\right] \\
& =\sqrt{I \gamma} \boldsymbol{A}\left[\begin{array}{l}
x_{1} \\
x_{2}
\end{array}\right]+\left[\begin{array}{l}
\sqrt{\lambda_{1}} n_{\mathrm{D}, 1} \\
\sqrt{\lambda_{2}} n_{\mathrm{D}, 2}
\end{array}\right]
\end{aligned}
$$

where

$$
\boldsymbol{A}=\left[\begin{array}{cc}
\sqrt{\lambda_{1}} h_{\mathrm{SD}} & 0 \\
\sqrt{\lambda_{2} v} h_{\mathrm{RD}} & \sqrt{\lambda_{2}} h_{\mathrm{SD}}
\end{array}\right] .
$$

In the following, we assume a frequency-flat block fading channel, the relay station has the perfect information of $h_{\mathrm{SR}}$, and the destination has the perfect information of $h_{\mathrm{SD}}$ and $h_{\mathrm{RD}}$. In order for the relay station to be able to decode the signal, the transmission rate in slot $1, R_{1}$, must satisfy

$$
\begin{aligned}
R_{1} & <\log _{2}\left(1+\frac{E_{\mathrm{SR}}\left|h_{\mathrm{SR}}\right|^{2}}{I_{1}}\right) \\
& =\log _{2}\left(1+\left|h_{\mathrm{SR}}\right|^{2} \mu \lambda_{1} \gamma\right) \triangleq R_{\text {relay }}^{\max } .
\end{aligned}
$$

In addition, in order to decode at the destination, the transmission rate in slot $i, R_{i}$, must satisfy

$$
\begin{aligned}
R_{1} & <\log _{2}\left(1+\|\boldsymbol{a}\|^{2} \gamma\right) \\
& =\log _{2}\left(1+\left|h_{\mathrm{SD}}\right|^{2} \lambda_{1} \gamma+\left|h_{\mathrm{RD}}\right|^{2} v \lambda_{2} \gamma\right) \triangleq R_{1}^{\max } \\
R_{2} & <\log _{2}\left(1+\frac{E_{\mathrm{SD}}\left|h_{\mathrm{SD}}\right|^{2}}{I_{2}}\right) \\
& =\log _{2}\left(1+\left|h_{\mathrm{SD}}\right|^{2} \lambda_{2} \gamma\right) \triangleq R_{2}^{\max }
\end{aligned}
$$

where $\boldsymbol{a}$ denote the first column of $\boldsymbol{A}$ and $\|\cdot\|$ is the Euclidean norm. In addition, since the relation between input and output (3) can be treated as a multiple-input multipleoutput (MIMO) channel, the sum rate $R_{1}+R_{2}$ must satisfy

$$
\begin{aligned}
R_{1}+R_{2}< & \log _{2} \operatorname{det}\left(\boldsymbol{I}_{2}+\boldsymbol{A} \boldsymbol{A}^{\mathrm{H}} \gamma\right) \\
= & \log _{2}\left[1+\left(\left|h_{\mathrm{RD}}\right|^{2} \gamma \lambda_{2}+\left|h_{\mathrm{SD}}\right|^{2}\left(\lambda_{1}+\lambda_{2}\right)\right) \gamma\right. \\
& \left.\quad+\left|h_{\mathrm{SD}}\right|^{4} \lambda_{1} \lambda_{2} \gamma^{2}\right] \triangleq R_{\text {total }}
\end{aligned}
$$

where $\boldsymbol{I}_{2}$ denotes the identity matrix of size 2 , rather than the interference power. Since the sum rate $R_{1}+R_{2}$ must satisfy the capacity for the receptions at both the relay station and the destination, the sum rate is constrained by

$$
R_{1}+R_{2}<\min \left\{R_{\text {total }}, R_{\text {relay }}^{\max }+R_{2}^{\max }\right\}
$$

(more details in [6]). Ergodic capacity as a function of ECIR $\gamma$ can be expressed as

$$
\begin{aligned}
& f_{(\mathrm{RD}, \mathrm{SR})}(\gamma)=\frac{1}{2} \min \left\{\mathrm { E } \left[\operatorname { l o g } _ { 2 } \left[1+\left(\left|h_{\mathrm{RD}}\right|^{2} v \lambda_{2}\right.\right.\right.\right. \\
& \left.\left.\left.+\left|h_{\mathrm{SD}}\right|^{2}\left(\lambda_{1}+\lambda_{2}\right)\right) \gamma+\left|h_{\mathrm{SD}}\right|^{4} \lambda_{1} \lambda_{2} \gamma^{2}\right]\right], \\
& \left.\quad \mathrm{E}\left[\log _{2}\left(1+\left|h_{\mathrm{SR}}\right|^{2} \mu \lambda_{1} \gamma\right)+\log _{2}\left(1+\left|h_{\mathrm{SD}}\right|^{2} \lambda_{2} \gamma\right)\right]\right\}
\end{aligned}
$$

where the factor $1 / 2$ represents two slots required for this scheme and $\mathrm{E}$ denotes the expectation operator.

\subsubsection{Cooperative Relaying (RD, R)}

Since the source does not transmit in slot 2, unlike the scheme (RD, SR), the received signals can be written as

$$
\begin{aligned}
{\left[\begin{array}{l}
\sqrt{\lambda_{1}} y_{\mathrm{D}, 1} \\
\sqrt{\lambda_{2}} y_{\mathrm{D}, 2}
\end{array}\right] } & =\left[\begin{array}{l}
\sqrt{E_{\mathrm{SD}} \lambda_{1}} h_{\mathrm{SD}} \\
\sqrt{E_{\mathrm{RD}} \lambda_{2}} h_{\mathrm{RD}}
\end{array}\right] x_{1}+\left[\begin{array}{l}
\sqrt{\lambda_{1}} n_{\mathrm{D}, 1} \\
\sqrt{\lambda_{2}} n_{\mathrm{D}, 2}
\end{array}\right] \\
& =\sqrt{I \gamma} \boldsymbol{a} x_{1}+\left[\begin{array}{l}
\sqrt{\lambda_{1}} n_{\mathrm{D}, 1} \\
\sqrt{\lambda_{2}} n_{\mathrm{D}, 2}
\end{array}\right] .
\end{aligned}
$$

The transmission rate $R_{1}$ has to satisfy the capacity constraint for the receptions at both the relay station and the destination as

$$
R_{1}<\min \left\{R_{\text {relay }}^{\max }, R_{1}^{\max }\right\} .
$$

Ergodic capacity can be expressed as

$$
\begin{aligned}
& f_{(\mathrm{RD}, \mathrm{R})}(\gamma)=\frac{1}{2} \min \left\{\mathrm{E}\left[\log _{2}\left(1+\left|h_{\mathrm{SR}}\right|^{2} \mu \lambda_{1} \gamma\right)\right],\right. \\
&\left.\mathrm{E}\left[\log _{2}\left(1+\left|h_{\mathrm{SD}}\right|^{2} \lambda_{1} \gamma+\left|h_{\mathrm{RD}}\right|^{2} \gamma \lambda_{2} \gamma\right)\right]\right\} .
\end{aligned}
$$




\subsubsection{Cooperative Relaying (R, SR)}

Since the destination does not receive in slot 1 , unlike the scheme (RD, SR), the received signal can be written as

$$
\begin{aligned}
\sqrt{\lambda_{2}} y_{\mathrm{D}, 2} & =\left[\begin{array}{ll}
\sqrt{E_{\mathrm{RD}} \lambda_{2}} h_{\mathrm{RD}} & \sqrt{E_{\mathrm{SD}} \lambda_{2}} h_{\mathrm{SD}}
\end{array}\right]\left[\begin{array}{l}
x_{1} \\
x_{2}
\end{array}\right]+\sqrt{\lambda_{2}} n_{\mathrm{D}, 2} \\
& =\sqrt{I \gamma} \boldsymbol{b}^{\mathrm{T}}\left[\begin{array}{l}
x_{1} \\
x_{2}
\end{array}\right]+\sqrt{\lambda_{2}} n_{\mathrm{D}, 2}
\end{aligned}
$$

where $\boldsymbol{b}^{T}$ is the second row of $\boldsymbol{A}$. Since this relation can be treated as a multiple-input single-output (MISO) channel, the sum rate $R_{1}+R_{2}$ must satisfy

$$
\begin{aligned}
R_{1}+R_{2} & <\log _{2}\left(1+\|b\|^{2} \gamma\right) \\
& =\log _{2}\left(1+\left|h_{\mathrm{SD}}\right|^{2} \lambda_{2} \gamma+\left|h_{\mathrm{RD}}\right|^{2} v \lambda_{2} \gamma\right) \triangleq R_{1}^{\max } .
\end{aligned}
$$

In addition, the sum rate must satisfy the capacity constraint for the reception at the relay station,

$$
R_{1}+R_{2}<\min \left\{R_{1}^{\max }, R_{\text {relay }}^{\max }+R_{2}^{\max }\right\} .
$$

Ergodic capacity can be expressed as

$$
\begin{array}{r}
f_{(\mathrm{R}, \mathrm{SR})}(\gamma)=\frac{1}{2} \min \left\{\mathrm{E}\left[\log _{2}\left(1+\left|h_{\mathrm{SD}}\right|^{2} \lambda_{2} \gamma+\left|h_{\mathrm{RD}}\right|^{2} v \lambda_{2} \gamma\right)\right],\right. \\
\left.\mathrm{E}\left[\log _{2}\left(1+\left|h_{\mathrm{SR}}\right|^{2} \mu \lambda_{1} \gamma\right)+\log _{2}\left(1+\left|h_{\mathrm{SD}}\right|^{2} \lambda_{2} \gamma\right)\right]\right\} .
\end{array}
$$

\subsubsection{Single-Hop Transmission}

The signal received by the destination in slot $i$ is given by

$$
y_{\mathrm{D}, i}=\sqrt{E_{\mathrm{SD}}} h_{\mathrm{SD}} x_{i}+n_{\mathrm{D}, i} .
$$

The transmission rate must satisfy

$$
R_{i}<\log _{2}\left(1+\frac{E_{\mathrm{SD}}\left|h_{\mathrm{SD}}\right|^{2}}{I}\right)
$$

Since for single-hop transmission there is no difference in capacity between both slots 1 and 2 , ergodic capacity can be expressed as

$$
f_{1}(\gamma)=\mathrm{E}\left[\log _{2}\left(1+\left|h_{\mathrm{SD}}\right|^{2} \gamma\right)\right] .
$$

\subsubsection{Two-Hop Transmission}

Two-hop transmission is different from cooperative relaying $(\mathrm{RD}, \mathrm{R})$ in that the destination does not receive in slot 1 . By eliminating the factor related to the S-D path in (13), we obtain the ergodic capacity of the two-hop channel

$$
\begin{aligned}
f_{2}(\gamma)=\frac{1}{2} \min \left\{\mathrm{E}\left[\log _{2}\left(1+\left|h_{\mathrm{SR}}\right|^{2} \mu \lambda_{1} \gamma\right)\right],\right. \\
\left.\mathrm{E}\left[\log _{2}\left(1+\left|h_{\mathrm{RD}}\right|^{2} v \lambda_{2} \gamma\right)\right]\right\} .
\end{aligned}
$$

Table 1 Ratio of interference power $\lambda_{1}, \lambda_{2}$.

\begin{tabular}{c|c|c}
\hline & 1, 2-hop, (RD, R) & (RD, SR), (R, SR) \\
\hline$\lambda_{1}$ & 1 & $2 / 3$ \\
$\lambda_{2}$ & 1 & $2 / 3$ \\
\hline
\end{tabular}

Table 2 Parameters used in the evaluation

\begin{tabular}{c|c}
\hline Parameters & Values \\
\hline Path loss exponent $\alpha$ & $2,3.5$ \\
Channel model & Rayleigh fading \\
Area environment & Interference-limited \\
\hline
\end{tabular}

\subsubsection{Number of Interference Signals}

There are two types of transmission in terms of the number of transmitters in a route. When using schemes (RD, $\mathrm{SR})$ or (R, SR) both the source and the relay station transmit in slot 2, as shown in Fig. 1(b), resulting in doubled interference signals compared to other situations in which either the source or the relay station transmit during a given time.

We then consider the resultant CIR when using schemes (RD, SR) or (R, SR). When assuming different transmissions are not synchronized, on average, in half of the routes both the source and the relay station transmit simultaneously, and in the other half of the routes either the source alone or the relay station alone transmits. Therefore, the number of interference signals is increased by a factor of $3 / 2$, and the received CIR in the (RD, SR) and (R, SR) is approximately $2 / 3$ of that in the other schemes. Table 1 summarizes the ratio of interference power $\lambda_{i}$ for each scheme.

\subsection{Numerical Results}

Table 2 summarizes the parameters used in the following evaluation. The complex channel gains $h_{\mathrm{SD}}, h_{\mathrm{SR}}$, and $h_{\mathrm{RD}}$ are assumed to be i.i.d. $C \mathcal{N}(0,1)$, i.e., Rayleigh fading. We assume path loss with the path loss exponent $\alpha$. For the sake of simplicity, the effect of shadowing is not considered. Since the interference-limited situation is assumed, the absolute value of transmit power does not have an impact on the received CIR. Thus, the transmit power is assumed to be the same for all stations. Note that it is not fair to compare these schemes in terms of power consumption.

Let $\left(x_{\mathrm{R}}, y_{\mathrm{R}}\right)$ denote the position of the relay station when assuming the positions of the source and the destination are $(0,0)$ and $(1,0)$. Unless otherwise specified, the relay station is assumed to be on the center point of a straight line segment from the source to the destination $\left(\left(x_{\mathrm{R}}, y_{\mathrm{R}}\right)=(0.5,0)\right.$, i.e. $\left.\mu=v=2^{\alpha}\right)$. While these assumptions are not necessarily realistic, they do enable us to gain insight into the design of cooperative relaying systems.

\subsubsection{Noise-Limited Environments}

For comparison, we first evaluate the $\mathrm{BE}$ of single-isolated communication, i.e., in noise-limited environments. The BE 


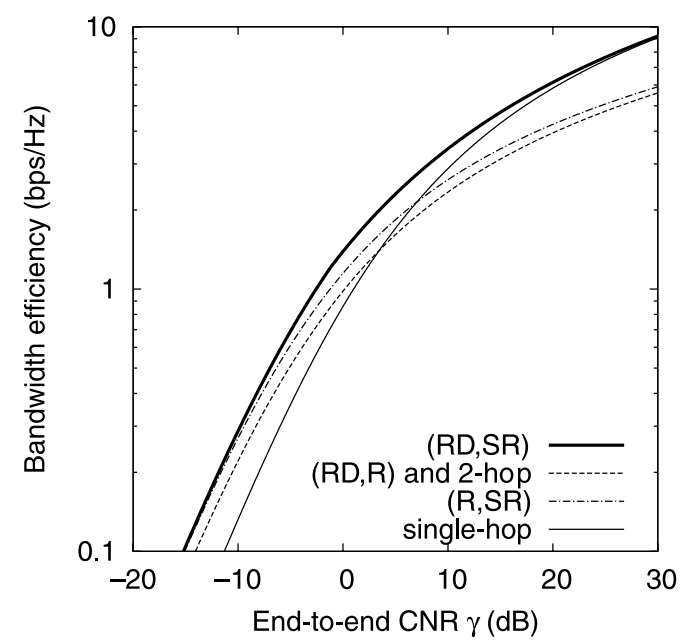

(a) $\alpha=2$.

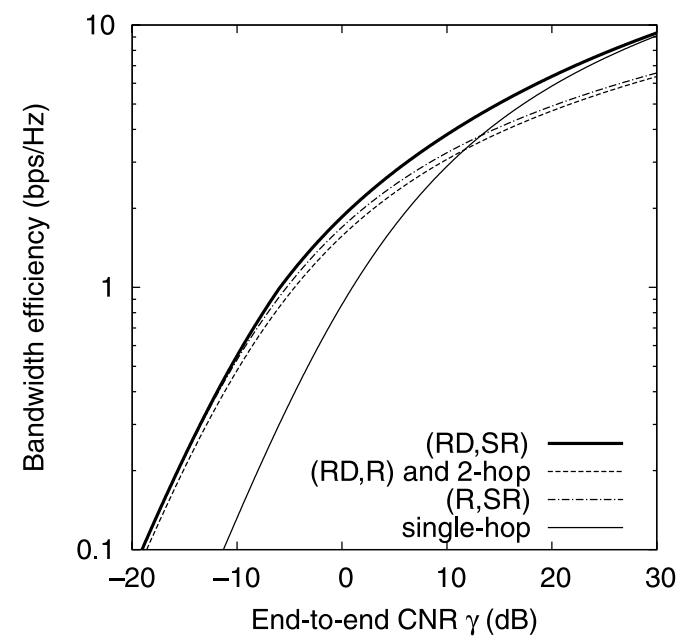

(b) $\alpha=3.5$.

Fig. 3 Bandwidth efficiency in noise-limited environments, $\left(x_{\mathrm{R}}, y_{\mathrm{R}}\right)=$ $(0.5,0)$.

in noise-limited environments can be obtained by substituting $\lambda_{1}=\lambda_{2}=1$ into Eqs. (10), (13), (16), (19), and (20), and replacing ECIR with the end-to-end carrier to noise ratio (ECNR), which is the received CNR at the destination regardless of whether the source and destination can communicate directly. The ECNR is a parameter that reflects both the transmit power per station and the end-to-end distance [13]. This substitution results in the same expression on ergodic capacity as described in [6] being obtained.

Figure 3 shows the BE in noise-limited environments. The (RD, SR) scheme always outperforms the other two cooperative relaying schemes because only this scheme has the same multiplexing gain as in MIMO, as shown in (8). In addition, (R, SR) always outperforms (RD, R). Note that these relationships are still preserved for any particular position of the relay station [6].

Let us next compare the (RD, SR) and non-cooperative single-hop transmission. Since $\lambda_{1}=\lambda_{2}=1$, and assuming $\mu\left|h_{\mathrm{SR}}\right|^{2}>\left|h_{\mathrm{SD}}\right|^{2}$ (recall that $\mu=2^{\alpha}$ and $\mathrm{E}\left[\left|h_{\mathrm{SR}}\right|^{2}\right]=$ $\left.\mathrm{E}\left[\left|h_{\mathrm{SD}}\right|^{2}\right]=1\right), f_{(\mathrm{RD}, \mathrm{SR})}(\gamma)$ satisfies

$$
\begin{gathered}
f_{(\mathrm{RD}, \mathrm{SR})}(\gamma) \\
=\frac{1}{2} \min \left\{\mathrm{E}\left[\log _{2}\left[1+\left(\left|h_{\mathrm{RD}}\right|^{2} v+2\left|h_{\mathrm{SD}}\right|^{2}\right) \gamma+\left|h_{\mathrm{SD}}\right|^{4} \gamma^{2}\right]\right],\right. \\
\left.\mathrm{E}\left[\log _{2}\left(1+\left|h_{\mathrm{SR}}\right|^{2} \mu \gamma\right)+\log _{2}\left(1+\left|h_{\mathrm{SD}}\right|^{2} \gamma\right)\right]\right\} \\
>\frac{1}{2} \min \left\{\mathrm{E}\left[\log _{2}\left(1+2\left|h_{\mathrm{SD}}\right|^{2} \gamma+\left|h_{\mathrm{SD}}\right|^{4} \gamma^{2}\right)\right],\right. \\
\left.\mathrm{E}\left[2 \log _{2}\left(1+\left|h_{\mathrm{SD}}\right|^{2} \gamma\right)\right]\right\} \\
=\mathrm{E}\left[\log _{2}\left(1+\left|h_{\mathrm{SD}}\right|^{2} \gamma\right)\right]=f_{1}(\gamma)
\end{gathered}
$$

which demonstrates that (RD, SR) outperforms single-hop transmission.

Two-hop transmission and (RD, R) have almost the same performance. This is because the first term dominates in Eq. (13) and both the first and second terms in Eq. (20) have approximately the same value as the dominant first term in Eq. (13) due to the assumption that the relay station is located at the center between the source and the destination.

\subsubsection{Interference-Limited Environments}

Figure 4 shows the BE in interference-limited environments. The technique to achieve the highest BE is changed from single-hop transmission to the (RD, SR) scheme and then to two-hop transmission and the (RD, R) scheme. Note that, in contrast to the noise-limited situation, (RD, SR) is not always superior to the other four schemes because of the reduction in each CIR value due to the simultaneous transmission of the source and the relay station in slot 2. On the other hand, (RD, R) or two-hop transmissions do not have an impact on the number of simultaneous transmissions compared to single-hop transmissions.

In noise-limited environments, the advantage of (RD, $\mathrm{SR})$ over $(\mathrm{RD}, \mathrm{R})$ and $(\mathrm{R}, \mathrm{SR})$ depends on neither ECNR nor the position of the relay station [6]. As described above, however, in interference-limited environments, the advantage depends on the ECIR and may also depend on the position of the relay station. Figure 5 shows the dependence of the technique achieving the highest $\mathrm{BE}$ on the position of the relay station $\left(x_{R}, y_{\mathrm{R}}\right)$. When the ECIR is high, singlehop transmission achieves the highest BE for most positions of the relay station, as shown in Fig. 5(a), and cooperative relaying achieves the highest $\mathrm{BE}$ only if a relay station near the destination can be used. Along with the reduction in the ECIR, the area where cooperative relaying achieves the highest BE is expanded, as shown in Figs. 5(b) to 5(d).

The effective area of cooperative relaying is approximately an overlapped area of two intersecting circles, the centers of which are located at the source and the destination. The effective area of $(\mathrm{RD}, \mathrm{R})$ is located nearer to the source than that of (RD, SR) and (R, SR) because when using $(\mathrm{RD}, \mathrm{R})$ and the relay station cannot decode the signal, the destination does not receive any signal in slot 2 , and thus the $\mathrm{BE}$ of $(\mathrm{RD}, \mathrm{R})$ is more highly dependent on the capacity of the link between the source and the relay station (the first term in (13)) compared to (RD, SR) and (R, SR). 


\subsection{Computer Simulation}

In order to verify the assumption concerning the interference described in Sects. 2.2 (recall that the ECIR's of all simultaneous communication pairs are greater than $\gamma$ and the
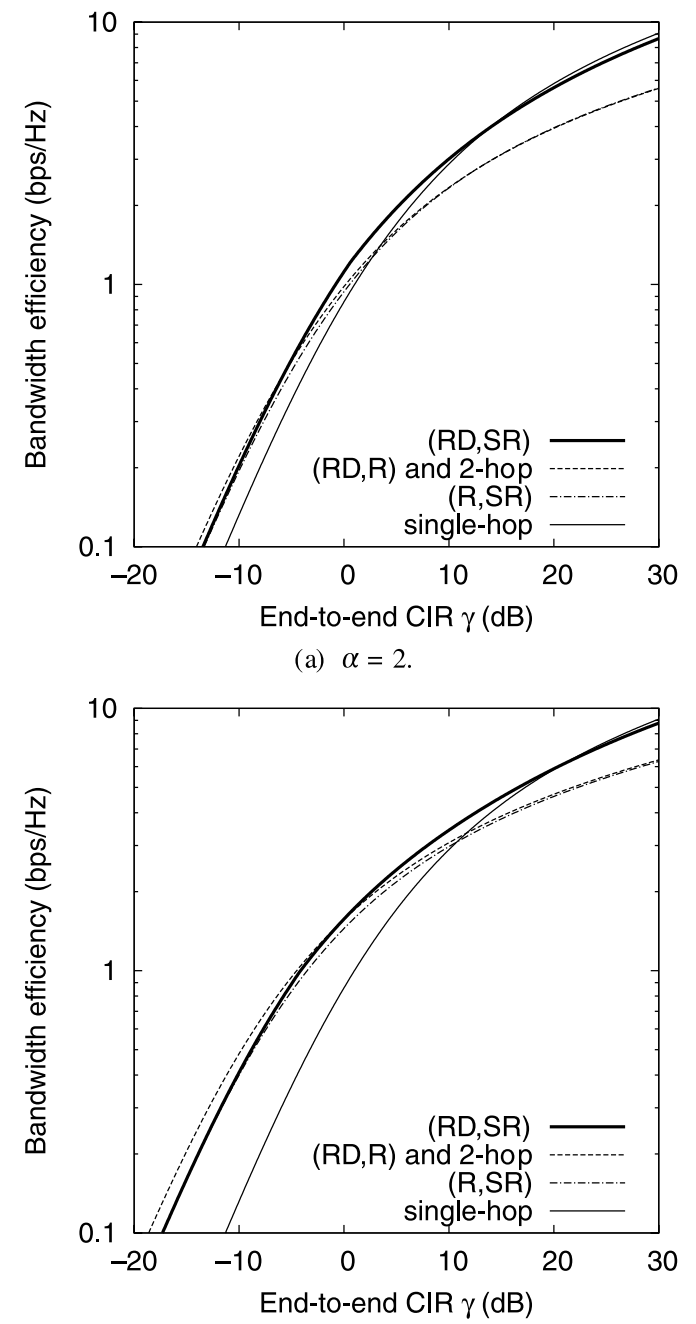

Fig. 4 Bandwidth efficiency in interference-limited environments, $\left(x_{\mathrm{R}}, y_{\mathrm{R}}\right)=(0.5,0)$.

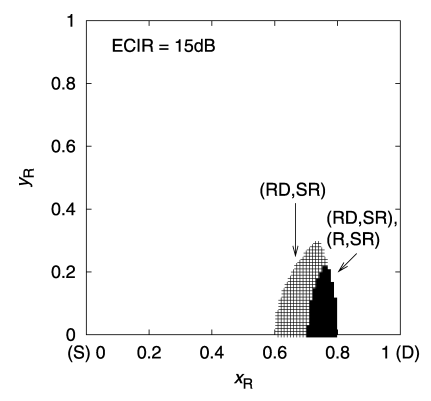

(a) $\mathrm{ECIR}=15 \mathrm{~dB}$. (a) $\alpha=2$.

(b) $\alpha=3.5$.

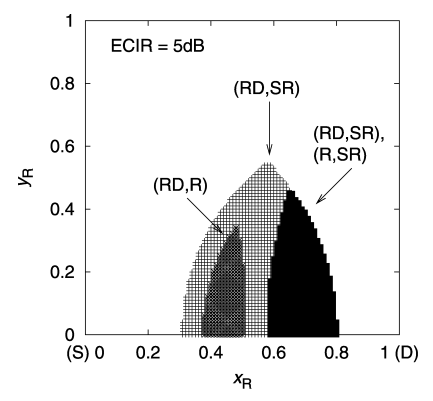

(b) $\mathrm{ECIR}=5 \mathrm{~dB}$.

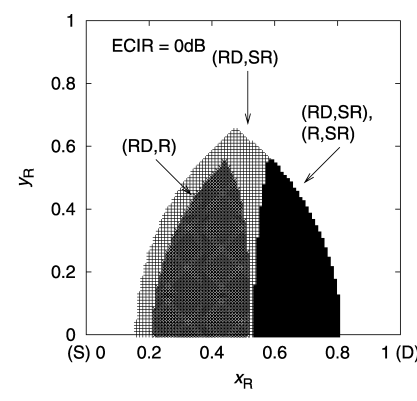

(c) $\mathrm{ECIR}=0 \mathrm{~dB}$. interference power from the source and that from the designated relay station are assumed to be the same) and 3.1.6 (recall that the received CIR of (RD, SR) and (R, SR) is approximately $2 / 3$ that of other cases), a computer simulation is used to evaluate the $\mathrm{BE}$ in random networks.

Table 3 summarizes the parameters used in the simulation. Source-destination pairs are uniformly and independently distributed in a two-dimensional square area and remain stationary throughout the evaluation. The distance between the source and the destination is set to be less than $500 \mathrm{~m}$.

The relay station is assumed to be located at the midpoint of a straight line segment that runs from the source to the destination because the purpose of this simulation is to verify the above assumptions in the analytical evaluation, and not to evaluate the impact of route selection. It is expected that similar results will be obtained according to the number of relay stations when there are uniformly and independently distributed relay stations and we select the relay station nearest to the center point.

Since the purpose of the present paper is to evaluate the impact of simultaneous communication on the capacity of cooperative relaying, each station pair is assumed to continuously communicate using the same power and same communication scheme only when the received carrier power at the source from already transmitting sources below a certain threshold. After the above procedure has been completed for all of the sources, the CIR's of S-R, R-D, and S-D are calculated, and based on these CIR's the ECIR and BE are evaluated. The threshold of this simple carrier sensing mechanism is used as a parameter to change the density of the simultaneous communication.

Figure 6 shows the BE. The order of performance is the same as that from numerical evaluations, as shown in Fig. 4(b). Thus the results validate the assumption used in

Table 3 Parameters used in the simulation.

\begin{tabular}{c|c}
\hline Parameters & Values \\
\hline Path loss exponent $\alpha$ & 3.5 \\
Channel model & Rayleigh fading \\
Area environment & Interference-limited \\
System area & $10 \mathrm{~km} \times 10 \mathrm{~km}$ \\
Number of communications & $<10^{4}$ \\
\hline
\end{tabular}

Fig.5 Most effective technique to achieve high bandwidth efficiency, $\alpha=3.5$. When the relay station is in a non-dotted area, single-hop transmission is the most effective technique. 


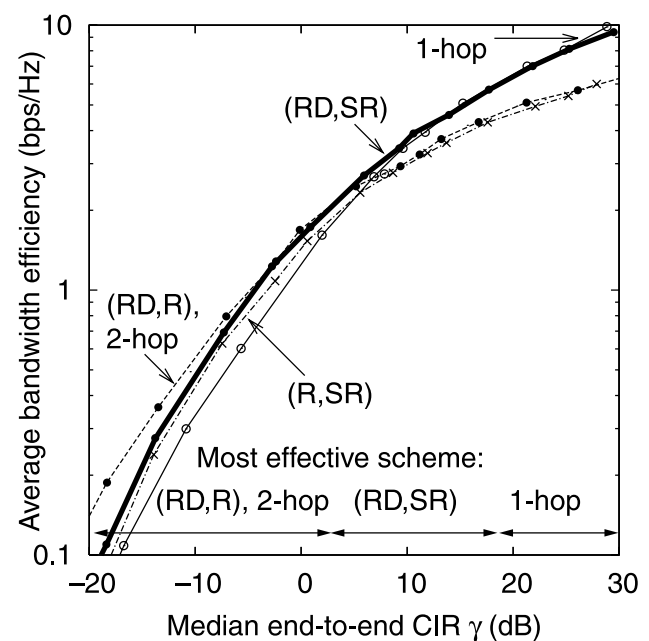

Fig. 6 Simulation result of bandwidth efficiency in interference-limited environments, $\left(x_{\mathrm{R}}, y_{\mathrm{R}}\right)=(0.5,0), \alpha=3.5$.

the numerical evaluation even for wireless networks with access control based on simple carrier sensing. Note that, since the simulation results are based on different assumptions than the results obtained by numerical evaluations, comparison of the absolute values of the average bandwidth efficiency is meaningless.

\section{Area Spectral Efficiency}

In radio communication systems, spatial channel reuse has a large impact on the ASE. In a single-hop transmission scenario, the decrease in the required CIR allows more simultaneous communications in a given area. However, each station should transmit at a lower rate. In addition, the introduction of two-hop transmission or cooperative relaying can decrease the required ECIR and allow more simultaneous communications.

In [13], we reported that the tradeoff between the ASE and $\mathrm{BE}$ of each communication is such that the use of multihop transmission can increase the ASE. Next, we investigate the impact on this tradeoff of the introduction of cooperative relaying. Under the assumptions described in Sect. 2.2, the ASE is the product of the BE and the density of the simultaneous communication.

\subsection{Analysis}

Recall that we have assumed that the ECIR's of all simultaneous communication pairs are greater than $\gamma$ and that the communication schemes of all station pairs are the same. Let $\rho(\gamma)$ denote the density of the simultaneous communication achieving the given ECIR $\gamma$.

For comparison, we also consider single-hop radio networks with QPSK. Let $\gamma_{\mathrm{QPSK}}$ denote the required carrier-tointerference plus noise ratio (CINR) for QPSK, which satisfies $\mathrm{BE}=2$. In order to satisfy $\gamma_{\mathrm{QPSK}}$, we assume that the distances between simultaneous communications need to be multiplied by $z$, while maintaining the distance between the source and the destination. The interference power is then reduced to roughly $1 / z^{\alpha}$ of the given network, so that the ECIR's of all transmissions satisfy the following condition:

$$
z^{\alpha} \gamma=\gamma_{\mathrm{QPSK}}
$$

Let $\rho_{\mathrm{QPSK}}$ be the density of the simultaneous communication, and let $\eta_{\mathrm{QPSK}}$ be the ASE of single-hop transmission with QPSK. The density of the simultaneous communication is reduced to roughly $1 / z^{2}$ of the given network

$$
\begin{aligned}
\rho_{\mathrm{QPSK}} & =\rho(\gamma) / z^{2} \\
& =\left(\frac{\gamma}{\gamma_{\mathrm{QPSK}}}\right)^{2 / \alpha} \rho(\gamma)
\end{aligned}
$$

Since the BE of QPSK is 2, the ASE of single-hop transmission with QPSK is expressed as the product of the BE and the density of the simultaneous communication

$$
\begin{aligned}
\eta_{\mathrm{QPSK}} & =2 \cdot \rho_{\mathrm{QPSK}} \\
& =2\left(\frac{\gamma}{\gamma_{\mathrm{QPSK}}}\right)^{2 / \alpha} \rho(\gamma) .
\end{aligned}
$$

Solving (23) for $\rho(\gamma)$ yields

$$
\rho(\gamma)=\frac{1}{2}\left(\frac{\gamma_{\mathrm{QPSK}}}{\gamma}\right)^{2 / \alpha} \eta_{\mathrm{QPSK}}
$$

The ASE of the given network is approximated by the product of $\rho(\gamma)$ and BE calculated in Sect. 3.

\subsection{Numerical Evaluations}

Figure 7 shows the ASE for the two different path loss exponents $\alpha$, where the absolute value of $\eta_{\mathrm{QPSK}}$ depends on the specific value of the path loss exponent $\alpha$. Therefore, there is no way to conduct a direct comparison between Figs. 7(a) and $7(\mathrm{~b})$.

Along with the decrease in ECIR, two-hop transmission outperforms single-hop transmission. Note that in terms of both BE in noise-limited environments in Fig. 3(b) and ASE in Fig. 7(b), the performances of two-hop and single-hop transmissions intersect at the same ECINR. However, since the use of (RD, SR) or (R, SR) increases the number of interference, the performances of (RD, SR) and other schemes do not intersect at exactly the same ECINR. Therefore, in order to evaluate the ASE of cooperative relaying, it is necessary to take into account the increase in the number of interference.

The tradeoff between ASE and BE [13] indicates that there is an achievable region of ASE and BE. Figure 8 shows that (RD, SR) expands the achievable region compared to single-hop and two-hop transmissions, where $0.5<\mathrm{BE}<$ $4(\alpha=2)$ and $2<\mathrm{BE}<6(\alpha=3.5)$. Note that, this tradeoff does not depend on the ECIR. Therefore, this type of cooperative relaying is truly effective for increasing the spectral efficiency of wireless systems. 


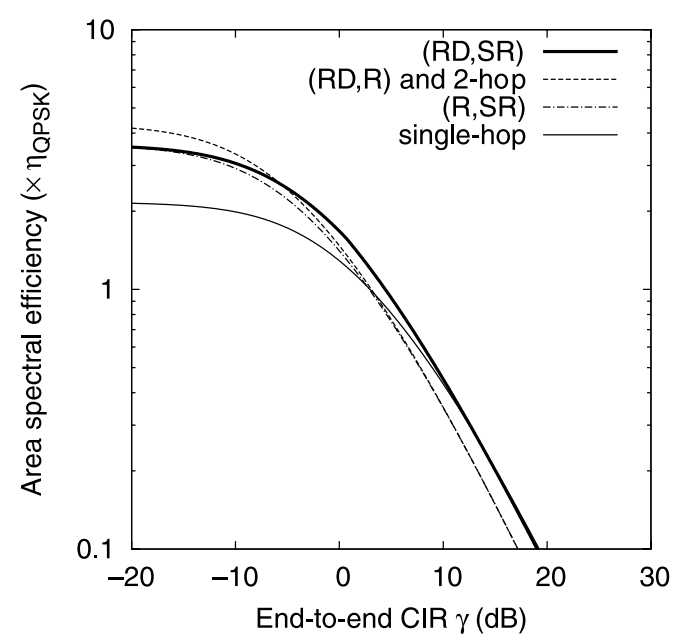

(a) $\alpha=2$.

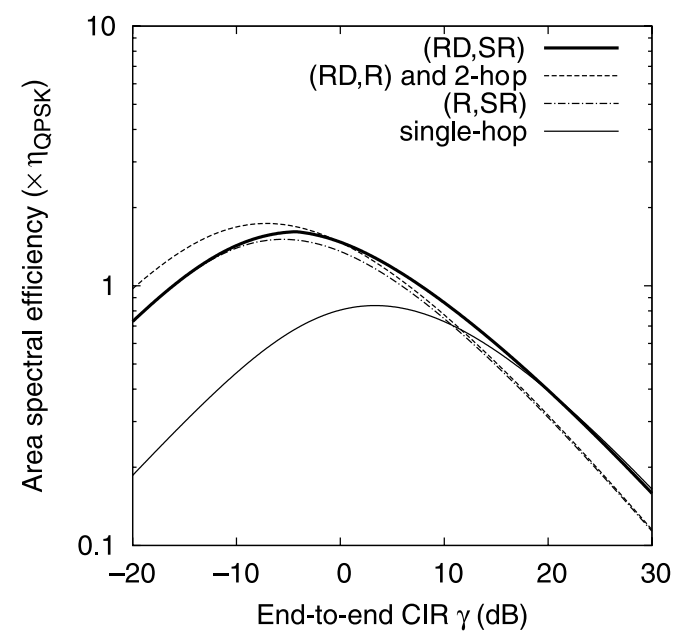

(b) $\alpha=3.5$.

Fig. 7 Area spectral efficiency in interference-limited environments, $\left(x_{\mathrm{R}}, y_{\mathrm{R}}\right)=(0.5,0)$.

\section{Concluding Remarks}

Assuming the most fundamental cooperative relaying, we have examined two types of spectral efficiency. First, we investigated the spectral efficiency per communication, which is referred to as the bandwidth efficiency. Numerical results revealed that the advantage of cooperative relaying in interference-limited environments depends on the density of the simultaneous transmission because of the increase in the number of interference signals, but does not depend on the density in noise-limited environments.

Next, we examined the impact of cooperative relaying on the tradeoff between bandwidth efficiency and overall spectral efficiency of wireless networks, referred to as the area spectral efficiency. The numerical results also reveal that the use of cooperative relaying expands the achievable region of area spectral efficiency and bandwidth efficiency compared to non-cooperative single-hop and two-hop transmissions.

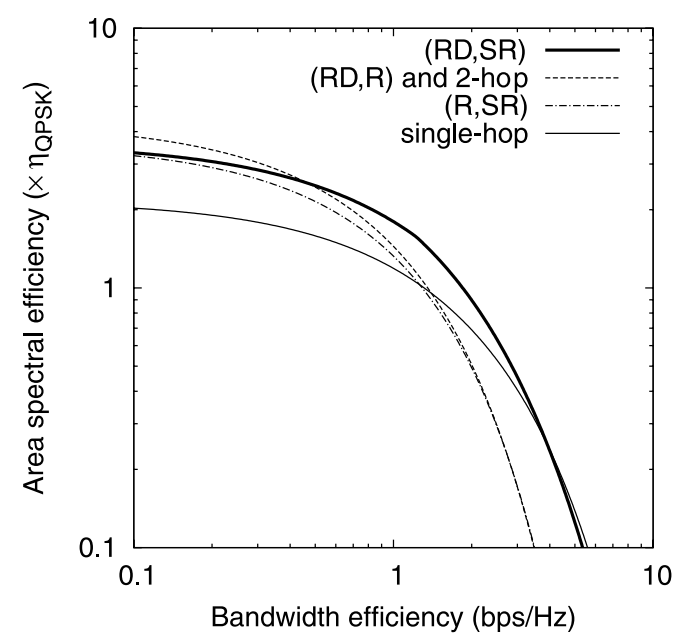

(a) $\alpha=2$.

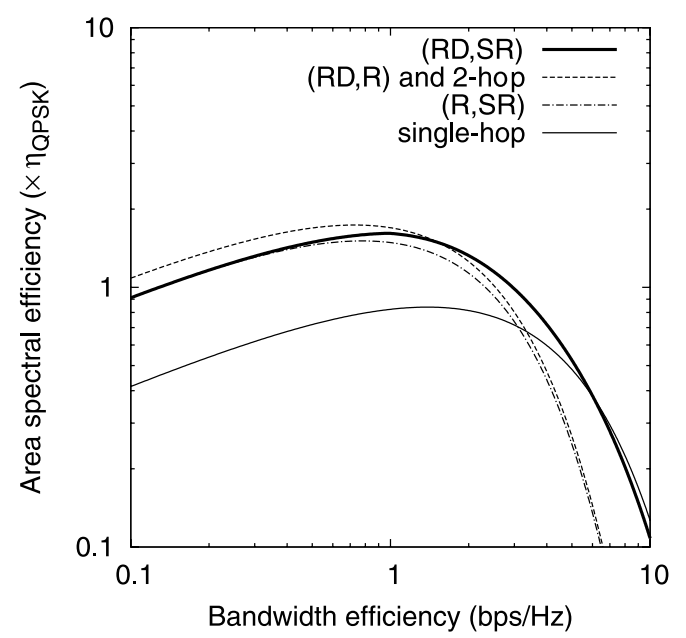

(b) $\alpha=3.5$.

Fig. 8 Area spectral efficiency vs. bandwidth efficiency in interference-limited environments, $\left(x_{\mathrm{R}}, y_{\mathrm{R}}\right)=(0.5,0)$.

We would like to emphasize that the purpose of the present paper is to clarify the circumstances under which cooperative relaying outperforms non-cooperative single-hop or two-hop transmission. We hope that the results presented in the present paper will provide insights that are useful in designing cooperative relaying systems. Further research is required for route selections in cooperative relaying.

\section{References}

[1] J. Weatherall and A. Jones, "Ubiquitous networks and their applications," IEEE Wireless Commun. Mag., vol.9, no.1, pp.18-29, Feb. 2002.

[2] R. Ramanathan and J. Redi, "A brief overview of ad hoc networks: Challenges and directions," IEEE Commun. Mag., vol.40, no.5, pp.20-22, May 2002.

[3] A. Sendonaris, E. Erkip, and B. Aazhang, "User cooperation diversity-part I: System description,” IEEE Trans. Commun., vol.51, no.11, pp.1927-1938, Nov. 2003.

[4] A. Sendonaris, E. Erkip, and B. Aazhang, "User cooperation diversity-part II: Implementation aspects and performance analysis," IEEE Trans. Commun., vol.51, no.11, pp.1939-1948, Nov. 2003. 
[5] J.N. Laneman, D.N.C. Tse, and G.W. Wornell, "Cooperative diversity in wireless networks: Efficient protocols and outage behavior," IEEE Trans. Inf. Theory, vol.50, no.12, pp.3062-3080, Dec. 2004.

[6] R.U. Nabar, H. Bölcskei, and F.W. Kneübuhler, "Fading relay channels: Performance limits and space-time signal design," IEEE J. Sel. Areas Commun., vol.22, no.6, pp.1099-1109, Aug. 2004.

[7] M. Haenggi, "Analysis and design of diversity schemes for ad hoc wireless networks," IEEE J. Sel. Areas Commun., vol.23, no.1, pp.19-27, Jan. 2005.

[8] T. Ohtsuki, "Performance analysis of statistical STBC cooperative diversity using binary sensors with observation noise," IEICE Trans. Commun., vol.E89-B, no.3, pp.970-973, March 2006.

[9] R. Haas and J.C. Belfiore, "Spectrum efficiency limits in mobile cellular systems," IEEE Trans. Veh. Technol., vol.45, no.1, pp.33-40, Feb. 1996.

[10] M.S. Alouini and A.J. Goldsmith, "Area spectral efficiency of cellular mobile radio systems," IEEE Trans. Veh. Technol., vol.48, no.4 pp.1047-1066, July 1999.

[11] K.J. Hole and G.E. Øien, "Spectral efficiency of adaptive coded modulation urban microcellular networks," IEEE Trans. Veh. Technol., vol.50, no.1, pp.205-222, Jan. 2001.

[12] S. Toumpis and A.J. Goldsmith, "Capacity regions for wireless ad hoc networks," IEEE Trans. Wireless Commun., vol.2, no.4, pp.736-748, July 2003.

[13] K. Yamamoto and S. Yoshida, "Tradeoff between area spectral efficiency and end-to-end throughput in rate-adaptive multihop radio networks," IEICE Trans. Commun., vol.E88-B, no.9, pp.3532-3540, Sept. 2005.

[14] J. Jun and M.L. Sichitiu, "Fairness and QoS in multihop wireless networks," Proc. IEEE VTC 2003-Fall, pp.6-9, Oct. 2003.

[15] V. Gambiroza, B. Sadeghi, and E.W. Knightly, "End-to-end performance and fairness in multihop wireless backhaul networks," Proc. MOBICOM 2004, pp.287-301, Sept.-Oct. 2004.

[16] R.U. Nabar and H. Bölcskei, "Space-time signal design for fading relay channels," Proc. IEEE GLOBECOM'03, vol.4, pp.1952-1956, Dec. 2003.

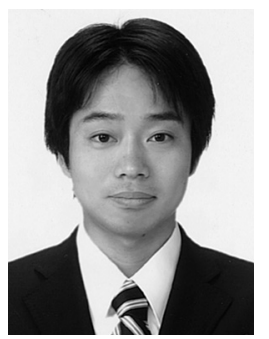

Koji Yamamoto received the B.E. degree in electrical and electronic engineering from Kyoto University in 2002, and the M.E. and Ph.D. degrees in informatics from Kyoto University in 2004 and 2005, respectively. Since 2005, he has been an assistant professor of the Graduate School of Informatics, Kyoto University. His research interests include cooperative multihop radio networks and distributed controls. $\mathrm{He}$ received the PIMRC 2004 Best Student Paper Award in 2004, the Ericsson Young Scientist Award in 2006, and the Young Researcher's Award from the IEICE of Japan in 2008 . He is a member of the IEEE.

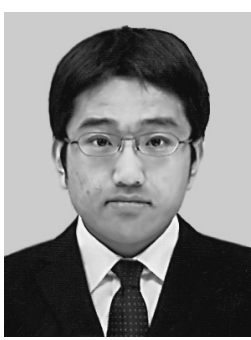

Hirofumi Maruyama received the B.E. degree in electrical and electronic engineering from Kyoto University in 2007. He is currently studying towards his M.E. degree at the Graduate School of Informatics, Kyoto University. His research interests includes multiantenna wireless systems.

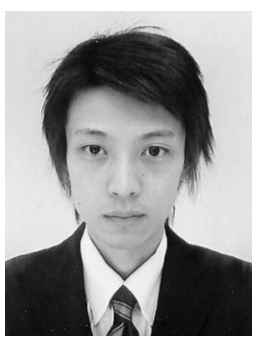

Takashi Shimizu received the B.E. degree in electronic engineering from Doshisha University in 2005, and the M.E. degree in informatics from Kyoto University in 2006. He is currently studying towards his Ph.D. degree at the Graduate School of Informatics, Kyoto University. His research interests includes receiver cooperative wireless networks.

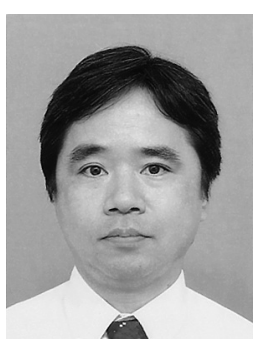

Hidekazu Murata received the B.S., M.S., and $\mathrm{Ph} . \mathrm{D}$. degrees in electronic engineering from Kyoto University, Kyoto, Japan, in 1991, 1993, and 2000, respectively. In 1993, he joined the Faculty of Engineering, Kyoto University. In 2002-2006, he was an associate professor of Tokyo Institute of Technology. Since 2006, he has been an associate professor of Kyoto University. His current research interests include signal processing and its hardware implementation, with particular application to multi-hop cooperative wireless networks. He received the Young Researcher's Award from the IEICE of Japan in 1997, the Ericsson Young Scientist Award in 2000, and the Young Scientists' Prize of the Commendation for Science and Technology by the Minister of Education, Culture, Sports, Science and Technology in 2006. He is a member of the IEEE and SITA.

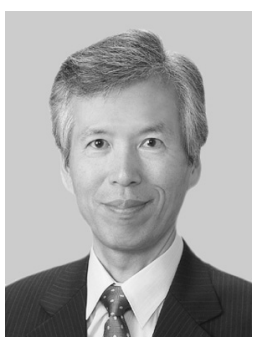

Susumu Yoshida received the B.E., M.E. and $\mathrm{Ph} . \mathrm{D}$. degrees all in electrical engineering from Kyoto University, Kyoto, Japan in 1971, 1973 and 1978, respectively. Since 1973, he has been with the Faculty of Engineering, Kyoto University and currently he is a full professor of the Graduate School of Informatics, Kyoto University. During the last 30 years, he has been mainly engaged in the research of wireless personal communications. His current research interest includes highly spectrally efficient wireless transmission techniques and wireless ad hoc networks. During 19901991, he was a visiting scholar at WINLAB, Rutgers University, U.S.A and Carleton University in Ottawa. He served as a TPC Chair of IEEE VTC 2000-Spring, Tokyo. He was a guest editor of IEEE J-SAC on Wireless Local Communications published in April and May 1996. He received the IEICE Achievement Award and Ericsson Telecommunication Award in 1993 and 2007, respectively. 